\title{
Verificação das relações de Rateaux pelo emprego de um inversor de freqüência ${ }^{1}$
}

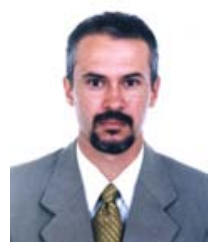

Aylton J. Alves ${ }^{2}$, Luiz F. C. de Oliveira ${ }^{3}$ \& Antônio M. de Oliveira ${ }^{4}$

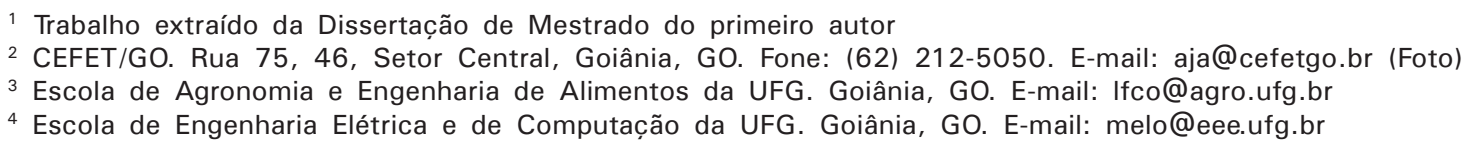

Protocolo $134-26 / 9 / 2001$

\begin{abstract}
Resumo: Este trabalho teve como objetivo verificar a veracidade das relações de Rateaux e a redução no consumo da potência consumida no bombeamento, quando se emprega o inversor de freqüência, o qual permite a variação de rotação do conjunto motobomba. Empregando-se o inversor de freqüência, a redução média da potência consumida foi de 40,7 e 75,0\% com a adoção da redução da rotação de 1800 para 1500 rotações min $^{-1}$ e de 1800 para 1100 rotações $\min ^{-1}$, respectivamente. Quando se avaliou a potência consumida usando-se essas mesmas relações, o erro médio na redução de potência foi de 1,33 e 2,00\% para as rotações min $^{-1}$ de 1500 e 1100, respectivamente, o que permite o emprego das relações para se estimar as grandezas altura manométrica, vazão e potência, a partir da curva característica obtida experimentalmente.
\end{abstract}

Palavras-chave: instalação de bombeamento, potência, curva característica

\section{Verification of the relationships of Rateaux utilizing a frequency invertor}

\begin{abstract}
OAbstract: This study had the objective to verify the relationships of Rateaux and the reduction of the power consumed during pumping when a frequency invertor is used it causes the variation of rotation of the motor-pump unit. Using a variable frequency drive, the observed average reduction in the power consumed was $40.7 \%$ with the reduction of the rotation from 1800 to 1500 rotations $\min ^{-1}$ and $75.0 \%$ for reduction from of 1800 to 1100 rotations $\mathrm{min}^{-1}$, respectively. When the power consumption was evaluated using the relationships of Rateaux, the mean error in the reduction of power was 1.33 and $2.00 \%$ for 1500 and 1100 rotations min $^{-1}$, respectively, which shows that the Rateaux relationships may be used for the estimation of manometric height, discharge and the power consumed from the characteristic curve obtained experimentally.
\end{abstract}

Key words: frequency variable drive, hydraulic pump, relationships of Rateaux

\section{INTRODUÇÃO}

Ao se projetar uma bomba hidráulica visa-se, especificamente, o recalque de determinada vazão em certa altura manométrica $(\mathrm{H})$ de modo a se obter o máximo rendimento. Entretanto, esta bomba poderá ser posta a recalcar vazões (Q) maiores ou menores que aquela para a qual foi projetada mudando, com a variação de $\mathrm{Q}$, a altura manométrica $(\mathrm{H})$, a potência necessária ao acionamento $(\mathrm{P})$ e o rendimento $(\mathrm{R})$ (Carvalho, 1977). Desta forma, cada bomba tem seu campo de aplicação em termos de grandeza, que interferem em seu funcionamento. A representação gráfica deste campo é chamada curva característica da bomba.

As bombas centrífugas são as mais empregadas em irrigação, apresentando a característica de reduzir o $\mathrm{H}$ com o aumento da $\mathrm{Q}$, o que requer maior $\mathrm{P}$, a ponto de poder sobrecarregar o motor. Variações na Q e H são muito comuns em sistemas de irrigação, principalmente em sistemas móveis, com movimentação manual ou mecânica.

Uma das formas de se ampliar o campo de aplicação de uma bomba para atender às variações na $\mathrm{H}$, é alterar a rotação de acionamento. Variando a rotação de acionamento, muda a curva característica da bomba, ou seja, a cada ponto H, Q a uma rotação n, corresponde, em semelhança mecânica, a um outro ponto H', Q' sob rotação n' (Azevedo Netto \& Alvarez 1982). As relações de Rateaux expressam as mudanças nas grandezas H, Q e P com a variação de n, ou seja:

$$
\frac{\mathrm{Q}^{\prime}}{\mathrm{Q}}=\frac{\mathrm{n}^{\prime}}{\mathrm{n}}, \quad \frac{\mathrm{H}^{\prime}}{\mathrm{H}}=\left(\frac{\mathrm{n}^{\prime}}{\mathrm{n}}\right)^{2} \text { e } \frac{\mathrm{P}^{\prime}}{\mathrm{P}}=\left(\frac{\mathrm{n}^{\prime}}{\mathrm{n}}\right)^{3}
$$


Mello et al. (1999) e Carvalho et al. (2000) comentam que um equipamento capaz de promover variações na rotação de acionamento com rapidez e eficiência, seguindo as relações de Rateaux, é o inversor de freqüência. Este equipamento trabalha alterando a freqüência da tensão aplicada ao motor, possibilitando controlar sua rotação. Campana et al. (2000) demonstraram que variações de $\mathrm{H}$ em sistemas de irrigação do tipo pivô central fazem com que os motores elétricos utilizados nos sistemas de bombeamento estejam, em grande parte do círculo irrigado, com índices de carregamento, rendimentos e fatores de potência inferiores aos nominais e que o uso de inversores de freqüência se apresenta como uma ferramenta útil para corrigir esses índices e aliviar o motor elétrico de cargas desnecessárias, resultando em uma redução no consumo de energia.

Em vista do exposto, este trabalho teve como objetivo verificar a veracidade das relações de Rateaux e a redução no consumo de energia, pelo emprego de inversor de freqüência em um sistema de bombeamento.

\section{MATERIAL E MÉTODOS}

Utilizou-se, neste trabalho, uma bomba centrífuga de pequeno porte, montado na microusina da Escola de Engenharia Elétrica da Universidade Federal de Goiás (EEEUFG) cuja potência do motor elétrico é de $3 \mathrm{HP}$, o qual, por sua vez foi interligado ao inversor de freqüência, permitindo a variação da rotação da motobomba (Fig. 1).

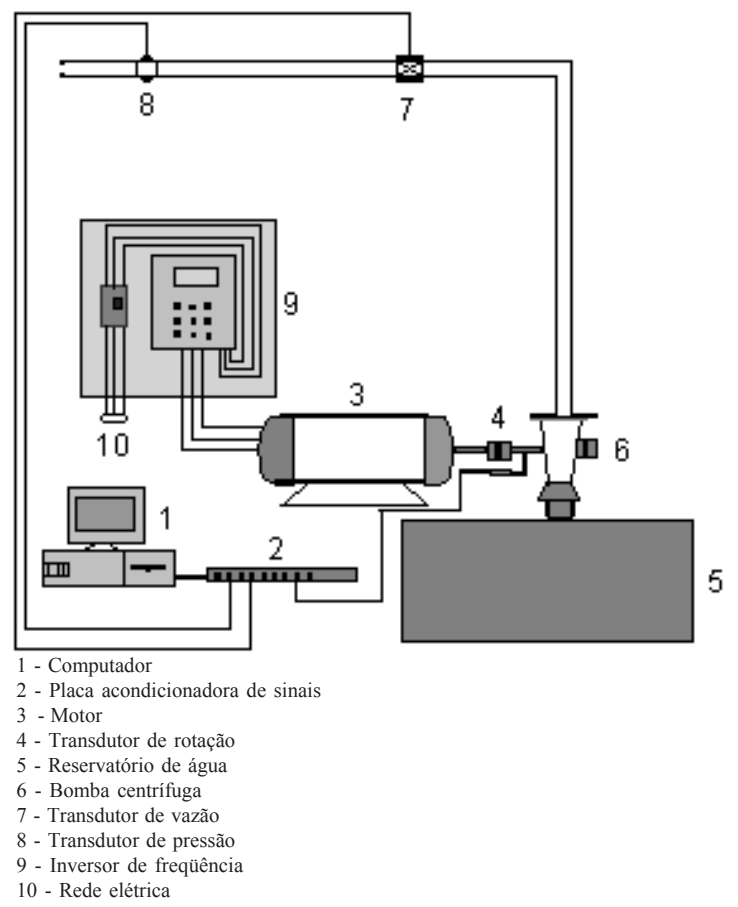

Figura 1. Desenho esquemático da bancada de ensaio

Na saída da tubulação de recalque foram instalados os transdutores de pressão e vazão e, no eixo do conjunto motobomba, um transdutor de rotação. Os transdutores foram interligados a uma placa acondicionadora de sinais (PAS) e esta a um computador.
As rotações empregadas neste trabalho foram 1800, 1500 e 1100 rotações min $^{-1}$, sendo a de 1800 rotações min $^{-1}$ a rotação tomada como nominal. Para cada rotação, variou a vazão recalcada pela abertura de uma válvula de gaveta. Os valores de Q e H, para cada rotação e posição da válvula de gaveta, foram armazenados na PAS, o que permitiu o traçado das curvas características.

Empregando-se as relações de Rateaux, calcularam-se as grandezas H, Q e P para n igual a 1500 e 1100 rotações min $^{-1}$, a partir da rotação nominal. Com os dados obtidos no experimento, verificou-se a veracidade das relações de Rateaux e a redução da potência consumida com a variação da rotação.

\section{RESULTADOS E DISCUSSÃO}

A Figura 2 apresenta as curvas características para as diferentes rotações e as calculadas, empregando-se as relações de Rateaux, com base na rotação nominal. Com auxílio da planilha Excel, foram ajustados, segundo as sugestões de Arens \& Porto (1989) e Yanagi Júnior (1997), polinômios de terceiro e segundo graus para as curvas $\mathrm{H} \times \mathrm{Q}$ e $\mathrm{P} \times \mathrm{Q}$, respectivamente (Tabelas 1 e 2). Observa-se, na Figura 2, o bom ajuste dos polinômios aos dados observados e calculados empregando-se as relações de Rateaux, com coeficientes de determinação $\left(\mathrm{R}^{2}\right)$ todos acima de 0,99 (Tabela 1$)$. As curvas obtidas pelo emprego das relações de Rateaux ficaram bem próximas das experimentais permitindo, assim, a utilização dessas relações no traçado das curvas características da bomba para diferentes rotações, a partir de uma curva experimental.

Tabela 1. Polinômios ajustados para as curvas altura manométrica $(H)$ x vazão $(\mathrm{Q})$ e potência $(\mathrm{P})$ x vazão $(\mathrm{Q})$ para as rotações de 1800,1500 e 1100 rotações min $^{-1}$

\begin{tabular}{ccc}
$\begin{array}{c}\text { Rotação } \\
\left(\text { rotações } \text { min }^{-1}\right)\end{array}$ & $\begin{array}{c}\text { Altura Manométrica } \\
(\mathrm{kPa})\end{array}$ & $\mathrm{R}^{2}$ \\
\hline A. H x Q & & \\
$1800^{*}$ & $\mathrm{H}=383,2-15,9 \mathrm{Q}+1,30 \mathrm{Q}^{2}-0,41 \mathrm{Q}^{3}$ & 0,9994 \\
$150 *^{*}$ & $\mathrm{H}=265,2-13,2 \mathrm{Q}+0,90 \mathrm{Q}^{2}-0,24 \mathrm{Q}^{3}$ & 0,9999 \\
$1100^{*}$ & $\mathrm{H}=134,6-9,30 \mathrm{Q}+0,30 \mathrm{Q}^{2}-0,23 \mathrm{Q}^{3}$ & 0,9998 \\
$1500^{* *}$ & $\mathrm{H}=266,1-13,2 \mathrm{Q}+1,29 \mathrm{Q}^{2}-0,50 \mathrm{Q}^{3}$ & 0,9914 \\
$1100^{* *}$ & $\mathrm{H}=143,1-9,70 \mathrm{Q}+1,29 \mathrm{Q}^{2}-0,70 \mathrm{Q}^{3}$ & 0,9994 \\
\hline B. P x Q & & \\
$1800^{*}$ & $\mathrm{P}=1,9233+0,0973 \mathrm{Q}+0,0008 \mathrm{Q}^{2}$ & 0,9982 \\
$1500^{*}$ & $\mathrm{P}=1,1072+0,0765 \mathrm{Q}-0,0003 \mathrm{Q}^{2}$ & 0,9984 \\
$1100^{*}$ & $\mathrm{P}=0,4524+0,0429 \mathrm{Q}-0,0006 \mathrm{Q}^{2}$ & 0,9982 \\
$1500^{* *}$ & $\mathrm{P}=1,1149+0,0563 \mathrm{Q}+0,0005 \mathrm{Q}^{2}$ & 0,9989 \\
$1100^{* *}$ & $\mathrm{P}=0,4397+0,0222 \mathrm{Q}+0,0002 \mathrm{Q}^{2}$ & 0,9950 \\
\hline
\end{tabular}

A Tabela 2 apresenta o comparativo da redução de potência consumida, medida e calculada pelas relações de Rateaux, para as rotações de 1500 e 1100 rotações minn $^{-1}$ e vazões de 1,2 e $4 \mathrm{~m}^{3} \mathrm{~h}^{-1}$, tomando-se como base a rotação nominal. A redução média de P com a adoção da redução da rotação de 1800 para 1500 rotações $\min ^{-1}$ e de 1800 para 1100 rotações $\min ^{-1}$, foi de $40,7 \mathrm{e}$ $75,0 \%$ baseados nos dados experimentais, e de 42,0 e $77,0 \%$ 

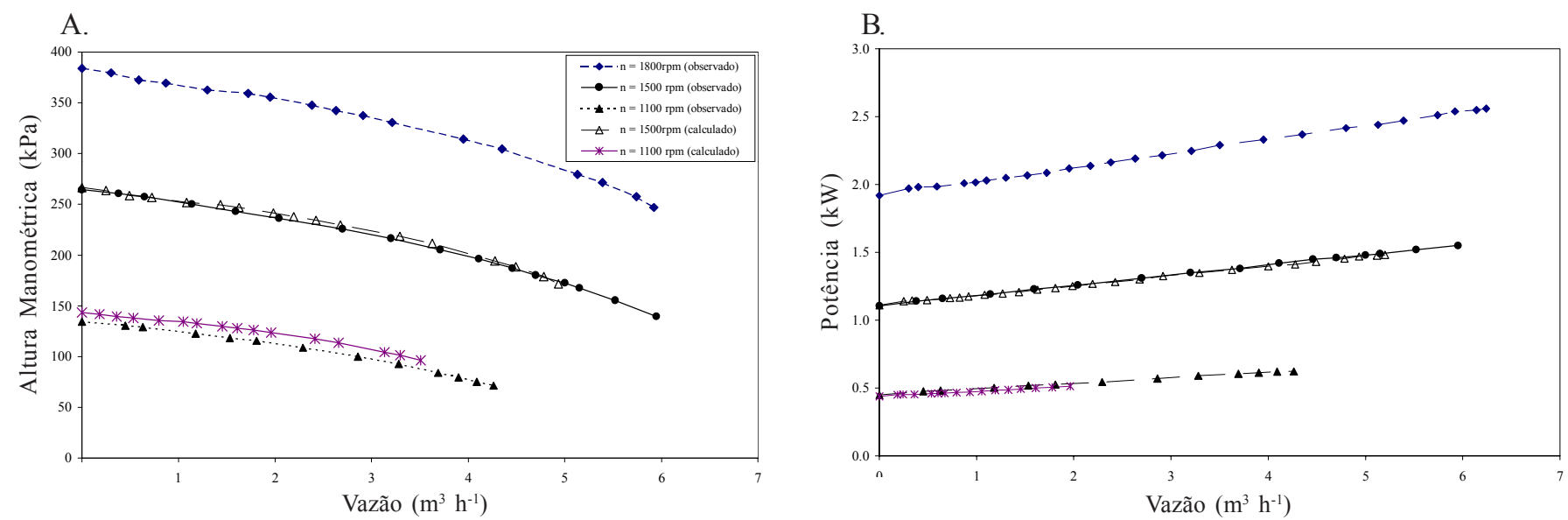

Figura 2. Curvas características de altura manométrica x vazão (A) e potência x vazão (B) observadas e calculadas para as diferentes rotações empregadas

Tabela 2. Comparativo da redução de potência consumida para as rotações de 1500 e 1100 rotações min ${ }^{-1}$ e vazões de 1,2 e $4 \mathrm{~m}^{3} \mathrm{~h}^{-1}$

\begin{tabular}{|c|c|c|c|c|c|c|c|c|c|}
\hline \multirow{3}{*}{$\begin{array}{c}\text { Vazão } \\
\left(\mathrm{m}^{3} \mathrm{~h}^{-1}\right)\end{array}$} & \multicolumn{9}{|c|}{ Rotação (rotações $\min ^{-1}$ ) } \\
\hline & \multirow{2}{*}{$\frac{1800}{\mathrm{P}_{\mathrm{obs}}(\mathrm{W})}$} & \multicolumn{4}{|c|}{1500} & \multicolumn{4}{|c|}{1100} \\
\hline & & $\mathrm{P}_{\text {obs }}(\mathrm{W})$ & $\Delta \mathrm{P} \%$ & $\mathrm{P}_{\text {Rateaux }}(\mathrm{W})$ & $\Delta \mathrm{P} \%$ & $\mathrm{P}_{\mathrm{obs}}(\mathrm{W})$ & $\Delta \mathrm{P} \%$ & $\mathrm{P}_{\text {Rateaux }}(\mathrm{W})$ & $\Delta \mathrm{P} \%$ \\
\hline 1 & 2,02 & 1,18 & 42,0 & 1,17 & 42,0 & 0,49 & 76,0 & 0,46 & 77,0 \\
\hline 2 & 2,12 & 1,26 & 41,0 & 1,23 & 42,0 & 0,54 & 75,0 & 0,48 & 77,0 \\
\hline 4 & 2,33 & 1,41 & 39,0 & 1,35 & 42,0 & 0,61 & 74,0 & 0,53 & 77,0 \\
\hline \multicolumn{2}{|c|}{ Redução de $\mathrm{P}_{\text {média }}(\%)$} & & 40,7 & & 42,0 & & 75,0 & & 77,0 \\
\hline \multicolumn{2}{|l|}{ Erro $_{\text {Médio }}(\%)$} & & \multicolumn{3}{|c|}{1,33} & \multicolumn{4}{|c|}{2,00} \\
\hline
\end{tabular}

com base nos dados obtidos nessas relações, respectivamente (Tabela 2). Ao se adotar as relações de Rateaux com a finalidade de se obter as curvas características da bomba para as diferentes rotações, ao invés de obtê-las com base em valores observados, isto acarretou em erro médio da $\mathrm{P}$, de 1,33 e 2,00\% para n igual a 1500 e 1100 rotações min $^{-1}$, respectivamente. Os erros médios de P são atribuídos ao fato de se ter levado em consideração a alteração no rendimento da bomba para os diferentes valores de vazão.

\section{CONCLUSÕES}

1. Empregando-se o inversor de freqüência, contatou-se que a redução média da potência consumida foi de foi 40,7 e $75,0 \%$, com a adoção da redução da rotação de 1800 para 1500 e 1100 rotações min $^{-1}$, respectivamente.

2. Quando se avaliou a potência consumida empregandose as relações de Rateaux, o erro médio na redução de $\mathrm{P}$ foi de 1,33 e $2,00 \%$ para as rotações de 1500 e 1100 rotações min $^{-1}$, respectivamente, o que permite o emprego dessas relações para se estimar as grandezas altura manométrica, vazão e potência a partir da curva característica obtida de forma experimental.

\section{LITERATURA CITADA}

Arens, H. G.; Porto, R. M. Para o desempenho de bombas. Revista DAE, São Paulo, v.49, n.11-14, 1989.

Azevedo Netto, J. M.; Alvarez, G. A. Manual de hidráulica. São Paulo: Edgard Blücher, 1982, 335p.

Campana, S. Racionalização do uso da energia elétrica em sistemas de irrigação tipos pivô central e aspersão convencional. Viçosa: UFV, 2000, 108p. Dissertação Mestrado

Carvalho, D. F. Instalações elevatórias: bombas. Belo Horizonte: IPUC, 1977, 355p.

Carvalho, J. A.; Mello, C. R.; Braga Júnior, R. A.; Reinato, C. H.; Santana, M. J. Utilização do inversor de freqüência em sistemas de irrigação para controle de vazão. Revista Brasileira de Engenharia Agrícola e Ambiental, Campina Grande, v.4, n.1, p.51-56, 2000.

Mello, C. R.; Carvalho, J. A.; Braga Júnior, R. A.; Reinato, C. H.; Santana, M. J. Economia de energia em instalação de bombeamento com o uso de inversor de freqüência. Engenharia Agrícola, Jaboticabal, v.18, n.4, p.78-88, 1999.

Yanagi Jr., T.; Ferreira, E.; Colombo, A.; Mello, C. R. Método matemático para a estimativa analítica das curvas de desempenho de bombas centrífugas. Revista Ciência e Agrotecnologia, Lavras, v.21, n.4, p.484-490, 1997. 